\title{
PERSPECTIVAS PARA UNA FILOSOFÍA DE LA TÉCNICA
}

Hans POSER

Universidad Técnica de Berlin

\section{1. ¿Técnica-Apocalipsis?}

En su incompleta utopía Nova Atlantis, escrita hacia 1624, Francis Bacon pone en boca de los ficticios habitantes de la isla la descripción de prodigios técnicos obtenidos mediante el dominio de la naturaleza merced a la ciencia natural aplicada. En aquella época la técnica estaba muy lejos de poder apoyarse sobre la ciencia o de ser ella misma una ciencia: era artesanía manual muy desarrollada. Pero hoy las ideas de Bacon de un mundo científico-técnico han sido hace ya tiempo alcanzadas y rebasadas por la realidad: la vida y la supervivencia humanas, la cultura y la forma de vida están cada vez más entretejidas con la técnica no sólo en los países industrializados; la técnica se ha convertido en un poder que determina la vida, y su simbiosis con la ciencia no es la causa menos importante de ello.

Cuando en la segunda mitad del siglo XIX la cientificación de la técnica cristalizó en obras de mecánica teórica, de cinemática teórica y de termodinámica técnica, siguió dominando la idea baconiana de que la técnica y la tecnología eran ciencias naturales aplicadas. Este punto de vista sigue vigente hoy, aunque desde hace tiempo se ha mostrado inadecuado desde dos perspectivas.

En primer lugar, el modelo baconiano transmite la impresión de que los artefactos técnicos son, como las leyes naturales sobre las que se basan, éticamente neutrales. Según esto, sólo con su utilización surgiría el problema de la responsabilidad. Hoy hemos corregido esta idea. Desde las conocidas consecuencias locales y globales de la técnica, la exigencia de una valoración previsora 
y adecuada de la técnica y una estimación de sus consecuencias ha ingresado en la conciencia pública hasta tal punto que la elocuente advertencia de Hans Jonas en El principio de responsabilidad pudo convertirse en un best-seller. Más aún: las visiones apocalípticas de una destrucción total de nuestro mundo a manos de la técnica implementada por la ciencia ocupan el lugar de las promesas de progreso. Los jinetes del Apocalipsis se llaman hoy agujero de ozono, catástrofe climática, contaminación radiactiva y overkill, y su cabalgada ha comenzado en Bhopal, Seveso, Chernobil e Hiroshima.

En segundo lugar, el modelo baconiano reduce la técnica a una imagen materialista de artefactos materiales inadecuada a la técnica de hoy y recubierta a su vez con los medios de una teoría de la ciencia orientada por la física.

A la influencia de la técnica sobre nuestro pensamiento y nuestra acción, nuestro saber y nuestro poder, una influencia que nunca fue tan poderosa como ahora, se enfrentan en el presente unas visiones apocalípticas que al mismo tiempo nos ciegan frente a la necesidad vital de la técnica. Tanto más urgente se hace emprender la reflexión sobre la técnica, que lleva una existencia a la sombra si se la compara con los ámbitos tradicionales de la filosofía, una reflexión que hay que entender como una tarea central y que conecta casi todas las cuestiones filosóficas. Ciertamente, las cuestiones de ética relacionadas con la técnica han pasado suscitar el interés público en los últimos años, pero ello no nos exime de la necesidad de indagar con la suficiente amplitud lo que constituye la esencia de la técnica y su dinámica. ¡De qué sirven los más hermosos principios de responsabilidad cuando pasan de largo frente a la cosa misma! De hecho, tampoco las obras que se esfuerzan por desarrollar en profundidad una ética de la técnica pueden pasarse sin sus propios análisis de la técnica (casi siempre desarrollados desde la perspectiva de la teoría de la acción) '. Por ello hay que reservar un puesto importante en la filosofía a la reflexión acerca de la técnica, pues sólo entonces podrá sustraerse al reproche de permanecer en su torre de marfil o de dedicarse a insignificantes tareas de filosofía de la ciencia; sólo de este modo podrá intervenir en

1 Valgan los siguientes ejemplos: Heiner HASTEDT, Aufklärung und Technik. Grundprobleme einer Ethik der Technik, Frankfurt a. M., Suhrkamp 1991; Christoph HuBIG, Technik-und Wissenschaftsethik. Ein Leitfaden, Berlin, Springer 1993; Thomas GIL, Demokratische Technikbewertung, Berlin, Berlin-Vlg. 1999. 
la discusión pública. Por eso en lo que sigue la ética de la técnica no ocupará el centro de nuestra atención, sino que éste corresponderá a las múltiples facetas de los problemas y al intento de ganar un punto de partida vinculante para una filosofía de la técnica.

\section{Elementos de la técnica y dificultades de una conexión conceptual}

Si el modelo de Bacon se queda corto con su cercanía a las ciencias, es preciso que nombremos aquellas características que diferencian esencialmente a un objeto técnicamente producido de cualquier objeto natural y exigen, con ello, someter la producción en tanto que acción desde el comienzo (frente a la tesis de la neutralidad) a principios éticos:

- La técnica es realización de ideas (Dessauer) ${ }^{2}$.

- La técnica se produce intencionalmente, es decir, con plena intención y orientada a ciertos fines. Este elemento de su desarrollo conceptual y de construcción debe encontrar correspondencia en el artefacto realizado y en el proceso realizado para poder diferenciar la técnica del arte en el sentido que hoy tiene esta palabra.

- La técnica es teleológica, pues ha de estar configurada de tal modo que el fin al que se tiende se alcance con ella como medio (de lo contrario ya no se trataría de técnica, sino de chatarra), y sólo del fin adquiere la técnica su sentido; y este fin es una forma de lo que está "al servicio del dominio creciente", por oposición al "desocultamiento a nuestro servicio", propio del arte (H. Beck) ${ }^{3}$.

- La técnica se orienta al cumplimiento de una función con vistas al fin que se persigue, no (como la ciencia) al conocimiento y la aproximación a la verdad. Por eso los científicos técnicos no piensan y argumentan con deducciones a partir de leyes naturales, sino con reglas,

2 «La técnica es un ser real que procede de ideas a través de la configuración finalística y la elaboración que parte de materiales dados en la naturaleza". F. DESSAUER, Streit um die Technik, Frankfurt a. M., Knecht 1956, pág. 234. Una definición semejante encontramos ya en Dessauer, Philosophie der Technik. Das Problem der Realisierung. Bonn, Cohen, 1927.

3 H. BECK, Kulturphilosophie der Technik, Trier, Spee, 1979, pág. 30. 
modelos y procesos, por ejemplo cómo un estado de cosas A puede conducir a un estado de cosas $\mathrm{B}^{4}$.

- La finalidad de un artefacto técnico es su esencia, independientemente de cómo se garantice el cumplimiento de la función: un reloj debe medir el tiempo, y esto es válido para los relojes de péndulo, los de cuerda, los de cuarzo, los de agua o los de sol 5 .

- La técnica es "el esfuerzo de ahorrarse esfuerzo" (Ortega y Gasset) ${ }^{6}$, o más en general: un desvio en la producción que ha de resultar provechoso (en un sentido muy amplio).

- Las producciones técnicas no son universales, sino singulares, y a menudo incluso absolutamente irrepetibles: piénsese en el proyecto de la presa de Wuhan, en China, en condiciones geológicas, ecológicas y sociales que no es posible encontrar en ningún otro lugar del mundo; y en la propia producción en cadena no se desarrolla "el automóvil en general", sino uno tal que sugiere en el comprador la idea de singularidad y al cual con mucha frecuencia se trata como un individuo. Con ello se hacen indispensables para la comprensión de la técnica los elementos de la hermenéutica.

- Los fines del desarrollo de una técnica específica así como el telos incorporado en el artefacto son fines externos y no, como en las ciencias empíricas, inmanentes. Así, un invento debe abrirse paso externamente como innovación, en el mercado y no entre los colegas especialistas.

- La dinámica de la técnica exige una interpretación adecuada, pues es (a diferencia de la dinámica natural) un producto del hombre, aunque pueda experimentarse como independiente de él. Si ha de poder ser dominada, se requiere una comprensión de la técnica que tienda un puente entre la acción técnica de los individuos y el fenómeno

4. K. KORNWACHS, "Vom Naturgesetz zur technologischen Regel - ein Beitrag zu einer Theorie der Technik", in: G. BANSE, K. FRIEDRICH (Hg.), Technik zwischen Erkenntnis und Gestaltung, Berlin, Sigma 1996, págs. 13-50

5 G. Simondon, Du mode d'existence des objets techniques, Paris, Aubier, 1958. Cf. también Paul Dumouchel, "Gilbert Simondon's Plea for a Philosophy of Technology", in: A. FEenberg, Alastair Hannay (eds.), Technology and the Politics of Knowledge, Bloomington, Indiana UP 1995, págs. 255-271.

6 J. ORTEGA y GASSET, Meditación de la técnica (1933). 
innegable de la dinámica propia del desarrollo técnico, que es o parece ser independiente de los individuos.

Sería ignorante afirmar que estas cuestiones no ha sido percibidas hasta ahora. Pero lo que falta en buen medida es su conexión en una filosofia de la técnica que posibilite al mismo tiempo una teoría de las ingenierías equivalente a la teoría de la ciencia. Para ello existen, junto al confundente paradigma de la física, al menos tres razones:

En primer lugar, la técnica es un elemento esencial de la cultura y ni se opone a ésta en tanto que civilización y ni es separable de ella como un parte (como podría decirse de una ciencia natural particular) porque la técnica, debido a su orientación finalística externa, se refiere siempre a toda la sociedad y se encuentra en interacción con ella. Sería deseable una filosofia de la cultura de la técnica que retomase la discusión que mantuvieron hasta los años treinta Julius Goldstein, José Ortega y Gasset, Ernst Cassirer, Ernst y Friedrich Georg Jünger y otros. La obra de Heinrich Beck ${ }^{7}$ no puede cubrir por sí sola este vacío, y la revista Technology and Culture no trata la problemática que precisamente anuncia su título.

En segundo lugar, la técnica es una necesidad vital. Desde Platón hasta Arnold Gehlen se ha subrayado una y otra vez que el hombre es un ser de carencias que necesita de ella ${ }^{8}$, pero sin desembocar en una nueva antropología de la técnica, una antropología que contenga además un modelo capaz de sustentar la intencionalidad colectiva. El comienzo que Hans Sachsse llevó a cabo hace dos décadas no ha sido proseguido ni en una ni en la otra dirección ${ }^{9}$.

En tercer lugar, la técnica es teleológica. Pero desde el Renacimiento la teleología se considera acientífica: en la teoría de la ciencia al uso no cuentan las explicaciones teleológicas, y casi lo mismo puede decirse de las explicaciones funcionales. Incluso las acciones humanas son reconstruidas en el silogismo práctico no como finalísticas, sino como intencionales. Pero una máquina no tiene intenciones, $y$ en cambio tiene fines incorporados.

7 H. BECK, Kulturphilosophie der Technik.

8 Platon, Protágoras, 320c-322a; A. Gehlen, "Der Mensh und die Technik", in: A. GEHLEN, Die Seele im technischen Zeitalter, Hamburg, Rowohlt 1957; reeditado en, A. GEHLEN, Anthropologische und sozialpsychologische Untersuchungen, Reinbeck, Rowohlt, 1986.

9 H. SACHSSE, Anthropologie der Technik. Ein Beitrag zur Stellung des Menschen in der Welt, Braunschweig, Vieweg 1978. 
Ciertamente, un cuchillo es adecuado para pelar patatas y para cometer un asesinato; $y$ un ordenador, al contrario que una maquina fabricada, no tiene ninguna finalidad fija, pero el ordenador está construido con vistas a ciertas posibilidades de trabajo cuyo esbozo se conoce (prefiguradas en el software que debe garantizarlas correspondiendo a los deseos del comprador). Hace falta, pues, una nueva teleología de la técnica que, si es posible transferir a esta cuestión la forma de pensar aristotélica de las posibilidades dinámicas, ha de ser también adecuada para proporcionar un modelo de la dinámica de la técnica. Como mostraremos en lo que sigue, semejante teleología de la técnica incluye también una hermenéutica de la técnica ${ }^{10}$, pues difícilmente pueden concebirse hoy los fines como elementos ónticos, sino que, antes bien, deben ser comprendidos como una interpretación que nosotros damos al artefacto técnico.

Sería deseable, en primer lugar, ordenar y disponer ambas tareas de un modo tal que se hiciera posible una conexión sistemática en la respuesta, tratándose, además, de un asunto que, si puede llevarse a término, ello sólo puede suceder gracias al esfuerzo conjunto de filósofos de diversa orientación temática y metodológica. Hay muchas voces que, con buenas razones, se manifiestan en contra de una respuesta unitaria y en favor de la consideración de la pluralidad de aspectos del problema y de formas de aproximación a él ${ }^{11}$. Precisamente a causa de las transformaciones que la técnica ha experimentado a lo largo de la historia, sería temerario admitir que pueda indagarse algo así como la esencia de la técnica en general; piénsese en el camino que conduce de la herramienta a máquina y de ésta a la técnica de grandes sistemas entrelazada con la ciencia; de los artefactos físicos a los biológicos pasando por los químicos; del fortalecimiento, prolongación y sustitución de órganos a la satisfacción de necesidades sociales culturalmente mediadas y de ésta al procesamiento de información, incluyendo el control de grandes sistemas. Realmente no podemos proponernos aquí establecer una síntesis, sino mostrar la filosofia de la técnica, en su significado propio y en toda la amplitud de sus cuestiones, como un desafío para la filosofía.

10 B. Irrgang ha dado un primer paso en esta dirección en N. A. CoronA, B. IRRGANG, Technik als Geschick? Geschichtsphilosophie der Technik bei Martin Heidegger. Eine Handlungstheoretische Entgegenung, Dettelbach, Röll 1999.

11 Por ejemplo, C. MITCHAM, Thinking through Technology. The Path between Engineering and Philosophy, Chicago, University of Chicago Press 1994 
Para esta clarificación son de gran ayuda tres modos de aproximación a la filosofía de la técnica que resultan ejemplares en su diversidad y que, cada uno a su modo, tienen todas legitimidad propia. Nos referimos a la interpretación analítica de Friedrich Rapp ${ }^{12}$, la de teoría de sistemas de Günter Ropohl ${ }^{13}$ y la de ontología fundamental en la línea de Martin Heidegger ${ }^{14}$. El primer modo intenta iluminar las dimensiones de la técnica partiendo de una aclaración analítica del concepto de técnica según una forma de pensamiento emparentada con la teoría de la ciencia. El segundo sitúa la realidad de la técnica en el centro y, adoptando una perspectiva propia de la ingeniería, intenta recoger en cuasi-diagramas sus relaciones con el hombre, la sociedad y el mundo de la vida, hasta donde pueden interpretarse en términos materialistas. El tercero, metafísico, parte del hombre y de su ser-ahí como ser en el mundo, al que la técnica sale al paso como "a-la-mano" o como "emplazado", desarrollada ante todo en el caso paradigmático de la herramienta y la máquina. Cada una de estas aproximaciones es fructífera a su manera, pero ninguna es capaz de tratar conjuntamente las cuestiones planteadas, sino sólo en aspectos parciales limitados ${ }^{15}$. De lado quedan en las tres aproximaciones tanto el componente intencional como el teleológico. Por eso es aconsejable buscar desde este punto una medio que permita revelar las perspectivas de filosofía de la técnica como perspectivas de un mismo problema.

\section{Materia, forma y finis: el problema ontológico}

Una reedición de la teleología aristotélica sería temeraria tras el tránsito por el pensamiento de la Edad Moderna, absolutamente hostil a la teleología. Pero

12 F. RaPP, Analytische Technikphilosophie, Freiburg-München, Alber 1978. Con todo, la última obra de este autor (Die Dynamik der modernen Welt. Eine Einfuihrung in die Technikphilosophie, Hamburg, Junius 1994) trasciende esta perspectiva.

13 G. ROPOHL, Eine Systemtheorie der Technik. Zur Grundlegung der Allgemeinen Technologie, München-Wien, Hanser 1979, págs. 197 y ss. (2a edición con el título Allgemeine Technologie, München, Hanser 1999, págs. 182 y ss.). La amplia obra de filosofía de la técnica de Ropohl ahonda en la diferenciación de esta perspectiva, pero en ocasiones va más allá de ella.

14 Resumida someramente por N. A. Corona en CORONA e IRRGANG, op. cit. Más detalladamente en G. SEUBOLD, Heideggers Analyse neuzeitlicher Technik, Freiburg-München, Alber 1986.

15 Con otros acentos y apoyándose parcialmente en otros autores, Langenegger ofrece una comparación de las diversas posiciones en D. LANGENEGGER, Gesamtdeutungen moderner Technik. Moscovici, Ropohl, Ellul, Heidegger. Eine interdiskursive Problemsicht, Würzburg, Königshausen \& Neumann 1990. 
lo que sí puede traerse aquí a colación es la transformación que ha experimentado la teleología. Recordemos para ello tres posiciones llamativas:

Leibniz se vio frente a la dificultad de armonizar la visión causal, y no finalística, del mundo de las cosas, tan existosa en el orden de la ciencia natural, con la creación divina de la máquina del mundo, creación conforme a un plan y, por tanto, finalística. La solución consistió en interpretar el mundo causal como fenómeno. Los fenómenos obedecen al principio de razón suficiente, en el sentido de un principio causal. Están plenamente fundados en el reino de las mónadas orgánicas, cuyos estados son producidos teleológicamente por una dinámica interna, siguiendo una ley individual. Esta relación del fenómeno y la mónada permite a su vez comprender todo ser vivo como una máquina, aunque, a diferencia de las máquinas producidas por el hombre, como una máquina de máquinas infinitamente compleja. La conexión exacta y preestablecida de las mónadas entre sí se funda por su parte en el hecho de ser realizada como parte del plan cósmico del mejor de los mundos posibles, es decir, como parte de un concepto monadológico de la teleología. Gracias a esta construcción, puede Leibniz hablar del reino de los fundamentos y las causas, por un lado, y del reino de los fines respecto de la elección divina, por otro lado. Ambos reinos se cruzan por todas partes, pero nunca se tocan, por usar la expresión de Leibniz. La máquina del mundo es causal y material, pero su auténtico fundamento de ser es finalístico e inmaterial. Con ello queda puesto de relieve un momento que pertenece a todo artefacto técnico, desde la herramienta hasta el gran sistema; un momento que para Friedrich Dessauer era el principal, cuando este autor consideraba la acción del ingeniero como la búsqueda de la forma ideal de solución y de su realización: la máquina es material, pero al mismo tiempo es la realización finalística de una idea.

Ambos autores, tanto Leibniz como Dessauer, vinculan a cierto platonismo su comprensión de la teleología y de la técnica: las finalidades se dan objetivamente en un mundo de ideas, y por eso se manifiestan en los artefactos en el caso de que lleguen a realizarse. Pero esto significaría que los fines son observables. Christian Wolff fue el primero en percatarse de que éste no puede ser el caso, sino que somos nosotros quienes introducimos los fines en las cosas con el supuesto de la existencia de un Dios creador o de un reino de las formas ideales de resolución, y acompañó su Física alemana con una Teleología alemana (los Pensamientos racionales de las intenciones de las cosas naturales). Kant hubo de radicalizar esta posición y separar sistemáticamente los fenómenos y la constitución de su estructura por las formas de pensamiento. La teleología se con- 
virtió en un asunto de la facultad de juzgar, en la que aparece también el concepto de "facultad de juzgar técnica», de forma sintomática de la nueva comprensión del problema ${ }^{16}$.

Ahora bien, no trataremos de ésta, como tampoco de eso que Johannes Rohbeck caracteriza como "facultad de juzgar tecnológica" ${ }^{17}$, porque este autor reserva este concepto exclusivamente para la reflexión sobre la responsabilidad de la técnica, y ello resultaría un punto de vista demasiado estrecho. La tesis que, con Kant, hay que asentar firmemente es la de que hay que construir una filosofía de la técnica partiendo del hombre, y no de un reino platónico de fines y formas ideales, pues somos nosotros los que valoramos algo (un estado de cosas existente), en una situación histórica concreta y con atributos de valor concretos e históricos, como algo necesitado de transformación y le oponemos otro estado de cosas que consideramos mejor, para después proponernos los medios, que asimismo hay que valorar, para pasar de un estado a otro. Las valoraciones y la voluntad humanas, el poder, el conocimiento y la creatividad humanos, en sus limitaciones históricas, se funden aquí y hacen que surjan los artefactos o los procesos técnicos. Más exactamente: no hacen que surjan, sino que surge algo que nosotros interpretamos como un medio técnico para el logro del fin al que tendemos. La técnica es, pues, un constructo interpretativo (Interpretationskonstrukt) en el sentido de Hans Lenks ${ }^{18}$.

La técnica en tanto idea realizada contiene un elemento material y otro formal en el sentido de Aristóteles, es decir, una materia, como aquello a partir de lo cual puede producirse un artefacto técnico, y una forma que al mismo tiempo es un finis, el cómo y el para-qué del artefacto. El a-partir-dequé procede de la naturaleza; el cómo y el para-qué se incorpora desde fuera, por parte del técnico (dejemos por el momento fuera de consideración que los materiales actuales mismos hace ya tiempo que no proceden de la naturaleza, sino que son productos técnicos, como aleaciones de metales, plásticos, cerámicas industriales, transuránicos, luz de laser polarizada, etc. Con todo, el punto de partida de estos materiales es siempre, en último término, algo

16 Cf., por ejemplo, la primera introducción a la Crítica del Juicio, XII.

17 J. ROHвECK, Technologische Urteilskraft. Zu einer Ethik technischen Handelns, Frankfurt a. M., Suhrkamp 1993.

18 H. LENK, "Handlung als Interpretationskonstrukt. Entwurf einer konstituenten-und beschreibungstheoretischen Handlungsphilosophiem, en H. LENK (Hg.), Handlungstheorien interdisziplinär, Bd. 2.1, München, Fink 1978, págs. 279-350. 
que se encuentra en la naturaleza). Es evidente que el material, en tanto sustrato, debe entrañar la posibilidad de realización, y por eso debe conocerlo el técnico que diseña y realiza el artefacto. La idea, en el sentido de Dessauer, es transformada, pues, partiendo de la materia por el técnico en el doble modo de conferirle el sentido de la forma y con vistas al finis. ¡Es transformada, pues, en una cosa material (o también en un proceso) que existe entonces con independencia del técnico y contiene una finalidad correspondiente a su intención como "finalidad intrínseca pura" ${ }^{19 !}$ No hay que olvidar que, pese al realismo ontológico de un técnico, también las propiedades atribuidas al sustrato, especialmente la posibilidad de realización, brotan de las perspectivas de saber y poder vinculadas al tiempo $y$, con ello, dependen de las condiciones de comprensión y de las atribuciones de sentido.

No hay artefactos aislados; la técnica moderna se caracteriza precisamente por su carácter sistemático. Este no es siempre explícita e inmediatamente perceptible, como el cableado de una red de información, sino que puede no llamar la atención, y ser objeto de ésta en sus elementos individuales, no como sistema total. Un automóvil, por ejemplo, se presenta como algo individual, pero está vinculado a carreteras, gasolineras, talleres mecánicos, sistemas de distribución de piezas de recambio, etc. cosas todas éstas que han de estar supuestas implícitamente en la idea del automóvil y de su materialización (no, ciertamente, como una carretera determinada, una gasolinera determinada, etc., sino sólo como una realidad fundamental de la que puede disponerse). Pero aquí reside una de las raíces de la dinámica de la técnica y de su comprensión: por una parte, el sistema depende de componentes parciales, pero por otra parte éstos no forman parte de una intención total, de modo que (y de esto se trataba) no se puede aceptar una finalidad a la que se tiende, al tiempo que se puede observar una interacción funcional de las partes. Este carácter sistemático de la técnica ha conducido en diversas ocasiones a comparar los sistemas técnicos con las ideas de Leibniz de la forma orgánica de los cuerpos en el sistema de las mónadas o, en general, con seres vivos. Este punto de vista es natural, pues el sistema técnico posee, de forma similar a un ser vivo, una cierta dinámica teleológica propia que indudablemente requiere una interpre-

19 J. LADRIĖRE, "The Technical Universe in an Ontological Perspective", ch. 2, in: $\mathrm{H}$. Lenk, Evandro AGAZZI and Paul DURBIN (eds.), Advances in the Philosophy of Technology: Proceedings of the International Academy of the Philosophy of Science, Karlsruhe, Germany, May 1997, in: Philosophy and Technology. Quaterly Electronic Journal 4 (1998). 
tación. Pero si se compara la teleología de los seres vivos con la de las máquinas, aparecen diferencias considerables:

- Los seres vivos tienen la finalidad de la conservación de sí y de la especie, la reproducción; en el caso de las máquinas no puede hablarse de algo así (en todo caso, no como característica general).

- La finalidad, en el caso de los seres vivos, está determinada intrínsecamente, en cierto sentido autónomamente y, si se da el caso, por la evolución; en el caso de las máquinas, es planeada e "incorporada" desde fuera, externamente, merced a la intencionalidad humana.

- La teleología humana es de una intencionalidad autónoma, la teleología de la máquina corresponde a nuestra intención, con la que construimos (perspectiva interna) e interpretamos (perspectiva externa) la máquina.

Es, pues, aconsejable no llevar la analogía demasiado lejos y no perder de vista las diferencias, aun cuando nos seduzca inmediatamente el pensamiento de ver las propiedades de los sistemas técnicos, que no son intencionales, según el modelo de las propiedades de los seres que actúan autónomamente.

En este punto surge una dificultad vinculada al concepto de artefacto. ¿Puede mantenerse este concepto (y su opuesto, que acabamos de esbozar) a la vista de la biotecnología? ¿Soy un artefacto, si se me implanta un marcapasos? ¿O me convertiría en uno, si se me implantase un tejido de células multipotentes cultivado artificialmente? ¿Es Dolly, la oveja clonada, un artefacto? ¿Y qué sucede con los tomates modificados mediante técnicas genéticas y con sus semillas? Es manifiesto que el concepto empleado hasta ahora es demasiado estrecho, y especialmente su limitación a las máquinas no hace justicia a las cosas, porque la biotecnología ha aparecido, con igualdad de derechos, junto a las tecnologías físico-químicas clásicas.

El mismo dilema se produce en los sistemas de procesamiento de información, que de forma no enteramente incorrecta han sido apostrofados de "herramientas de pensamiento" (Denkzeug) por oposición a las "herramientas» (Werkzeug). Resultaría extremadamente difícil tratar el procesamiento de información según un modelo técnico-mecánico, pues aunque el procesamiento es un proceso material (incluido en las biocomputadoras), y aunque el resultado (las masas de datos) tienen apariencia material porque aparecen en pantallas o en papel impreso, sin embargo es tan poco material como el contenido de un 
libro: piénsese en una demostración matemática desarrollada con una computadora. Más bien lo que se muestra sólo llega a ser el resultado intencional del proceso en conexión con una interpretación semántica.

Pero en ambos casos subsiste una diferencia decisiva frente a lo no técnico, una diferencia que ha guiado las reflexiones anteriores: se trata siempre de pasar, mediante una intervención conforme a un plan, de un estado de cosas dado $A$, a un estado de cosas deseado $B$, es decir, de realizar, siguiendo una intención, la representación de un fin en la materia, viva o inerte, interpretada o no interpretada. Pero esta determinación es, de nuevo, tan amplia que abarca e incluye en el concepto de la técnica los conceptos aristotélicos de poiesis y praxis, con el resultado de que también cortar el pelo o hacer música, en resumen, toda acción humana se convierte en técnica. Si se quiere dar a este concepto un sentido más restrictivo, ello sólo puede llevarse a cabo si se bosqueja con más exactitud de qué tipo de estado de cosas y de qué género de fines se trata en la transformación intencional. Como, desde hace poco tiempo, pueden patentarse los seres vivos modificados mediante técnicas genéticas, se revela una ampliación de nuestro antiguo concepto de poiesis que sólo a causa de su novedad nos resulta extraño. La necesidad de interpretar también como información lo que produce un sistema de procesamiento de información muestra una vez más que, si el ordenador, incluido su programa, se concibe como un artefacto, esto siempre supone interpretarlo al mismo tiempo como dotado de finalidad. El hecho de que esta finalidad sea ante todo una potencia, la posibilidad de procesamiento (algo sobre lo que volveremos más adelante) fortalece la línea directriz que aquí hemos trazado: la finalidad constituye la esencia de todo artefacto técnico y de todo proceso técnico. Interviene, como intencionalidad, en el proceso de invención y desarrollo, y en el uso se incorpora a una interpretación, lo que exige una materia y una forma correspondiente al finis. Aunque ambos ámbitos no se identifican nunca, se cruzan entre sí por todas partes.

Resumiendo, hay que retener que tras la ampliación gradual del concepto de la técnica desde el artefacto (las más de las veces, físico) al proceso (en principio, químico), ahora hay que recoger también en él el ámbito de los artefactos y procesos bioéticos, así como las "herramientas de pensamiento", es decir, los medios de transformación que sirven al procesamiento de información. Pero esto significa que revelar como demasiado estrechas las atribuciones ontológicas clásicas que siempre estuvieron vinculadas a la llamada técnica real. La delimitación de algo como "técnica" no puede tener éxito por lo que atañe 
exclusivamente a la ontología, sino que debe acertar con las finalidades y, por tanto, con la intencionalidad que se oculta tras ellas.

\section{Intención y finalidad: el problema hermenéutico}

Todo artefacto y todo proceso técnico se producen intencionalmente y están vinculados al cumplimiento de una función. A la intencionalidad de la poiesis corresponde la finalidad de lo producido. Pero a esto puede objetarse que las finalidades no se encuentran por ninguna parte, al menos en la naturaleza material; antes bien, lo que la técnica pone a su servicio sucede sólo causalmente. Si no fuese así, no sería posible la técnica, el cumplimiento regulado de funciones por medios técnicos para la consecución de fines dados. Y, siempre según esta objeción, en la naturaleza viva existen, junto a la causalidad, exclusivamente procesos evolutivos que excluyen igualmente toda finalidad. Los artefactos bioéticos, en el sentido de la biotecnología, sólo serían posibles en la medida en que estuviese dado un nexo fijo de reglas que garantizase el cumplimiento de la función. Realmente, todo esto es correcto; la técnica sin causalidad o regularidad es imposible. Pero estas regularidades mismas no forman parte todavía de la técnica; ha de añadirse la circunstancia de que las reglas sean aprovechadas para un dominio de la cosa «que las pone a su servicio" (Beck). Esto es precisamente lo que constituye la intencionalidad, del lado del técnico, y del lado del artefacto la finalidad. Si una verdura modificada mediante técnicas genéticas no resiste la putrefacción (fin inmediato) y no es, pues, comestible (fin mediato), apenas podremos llamarla ya verdura. Una máquina que no produce o no produce ya aquello a lo que apunta, sino, por ejemplo, sólo desperdicios, es una máquina defectuosa en un sentido simple, pero en un sentido más profundo ya no es la máquina en cuestión: se ha convertido en un sinsentido. Esto último, a su vez, deja claro que cuando se habla de una máquina, se la considera e interpreta atendiendo a su finalidad. Pero esta interpretación no se suma a otras propiedades, sino que su contenido teleológico constituye precisamente la determinación esencial del artefacto. Esto nos resulta tan evidente que el nexo sólo es reconocible cuando, en un caso individual, no está dado. Así, en el Museo Etnológico de Berlín, en la sección de Oceanía, hay un objeto cuya descripción reza: "Objeto de culto. Uso desconocido". Ahora bien, un objeto de culto no es un artefacto técnico en nuestro sentido, pero sí para una cultura organizada mediante los mitos y la magia, 
por lo cual el ejemplo muestra claramente que ya no queda absolutamente nada de la técnica mágica, sino solamente un objeto inventariado de madera o de hueso que se hurta a nuestra comprensión. Fue Heidegger quien vio esta relación cuando introdujo el concepto de lo "a-la-mano" y el del «armazón". Con ello, la comprensión de la técnica revela su dependencia específica de una hermenéutica adecuada a la cosa, cuyo objeto es el nexo de intencionalidad y atribución de un telos. El ejemplo permite asimismo reconocer que uno de los supuestos de esta hermenéutica consiste en una precomprensión históricamente fundada de la técnica y de sus fines. Si esto no llama usualmente nuestra atención, sucede así porque en las ingenierías, como en las ciencias naturales, la formación asegura una homogeneización de la precomprensión, que aparece como ahistórica y objetiva. Pero lo que sólo puede ser aprehendido en una hermenéutica de la tecnología va más allá de la comprensión de la intencionalidad y la finalidad: toda atribución de valores, desde el funcionamiento hasta los valores éticos, pasando por la seguridad, pertenece a ella, así como la comprensión de la situación particular de aplicación en su irrepetibilidad histórica. De este modo, un método que pertenece a las ciencias del espíritu ingresa en el tratamiento adecuado de la técnica.

Más arriba subrayábamos el carácter de sistema propio de la técnica actual. Pero de ello resultan dificultades especiales por lo que respecta tanto a la finalidad como a la intencionalidad; pues ni las finalidades individuales de las partes del sistema pueden ser tratadas aisladamente, sin incluir el entramado del sistema, ni la intencionalidad de los agentes individuales se suma convirtiéndose en una intencionalidad de grupo. Ciertamente, se han dado pasos hacia esto último ${ }^{20}$, pero falta toda referencia a la acción técnica. Por encima de todo, falta un modelo que sea capaz de reflejar el hecho y la causa de que el sistema conduzca a estados que no se propone ninguno de los agentes individuales, un fenómeno que se torna relevante sobre todo en relación con la cuestión de la responsabilidad en la valoración de la técnica y en la estimación de sus consecuencias.

No seguiremos aquí la problemática ética, pero para nuestro contexto es necesario tomar conciencia de lo siguiente: hasta ahora se definía, de forma simplifi-

20 Así John R. SEARLE, The Constitution of Social Reality, New York, Free Press 1995. También R. TuOmela, K. Miller, "We-Intentions", in: Philosophical Studies, 35 (1988), págs. 367 y ss. 
cadora, el "técnico" como aquel que diseña, construye y emplea un artefacto técnico. Pero desde el comienzo de la división del trabajo (y esto significa, por lo que respecta a las herramientas, desde la edad de piedra: piénsese en la extensión del comercio con herramientas de obsidiana en la cuenca del Mediterráneo) ya no podemos hablar en esos términos. Y esto vale tanto más para una sociedad completamente penetrada por la división del trabajo, con complejos sistemas técnicos: diseñadores, fabricantes y usuarios están completamente separados, y todos ellos se encuentran, por su parte, en redes de sistemas; puede decirse incluso que ya no se trata de individuos aislados, sino de sistemas de elevada complejidad: la sección del proyecto, la fabricación, el sistema de distribución, la empresa compradora, los usuarios, etc. Todos ellos encaran el elemento del sistema que han de diseñar, o fabricar, o utilizar con intenciones completamente diferentes que sólo tienen en común el hecho de que los diseñadores y fabricantes deben anticipar la intención del usuario (o del sistema de usuarios), es decir, su concepción de algún fin que él pueda alcanzar con ese elemento del sistema. Así (y esto es lo más importante) se convierte la finalidad del artefacto en un elemento de enlace también en este caso. Pero a esta finalidad se le sustrae al mismo tiempo su referencia a la intención de un individuo determinado, pues normalmente sólo puede anticiparse una muy abstracta concepción de una posible intención de un posible usuario. Por otro lado, esto tiene como consecuencia la intercambiabilidad no sólo del usuario, sino también del fabricante y del diseñador. La sociedad de masas refleja esta circunstancia en su homogeneización de las necesidades y de las concepciones de los fines individuales, la intercambiabilidad del trabajador así como la sustituibilidad del constructor por algún otro que posea el mismo saber y las mismas capacidades, niveladas mediante la formación profesional. Sólo es decisivo el funcionamiento global del sistema. Por eso no resulta sorprendente que Mumford hable de una "megamáquina” y Max Weber de una "máquina social»"

\section{Entre la factibilidad y la evolución: el problema de las formas de pensamiento}

Según Kant, las formas de pensamiento constituyen la forma mediante la cual constituimos los objetos a partir de lo material de la intuición.

21 L. Mumford, Mythos der Maschine, Frankfurt a. M., Suhrkamp 1977, pág. 220. 
Ciertamente, se querrá romper el vínculo de estas formas kantianas con la lógica aristotélica, pero la idea fundamental ha de retenerse: el sujeto de conocimiento es el que estructura y constituye los objetos de la experiencia mediante las formas, imprimiéndoles éstas. Whitehead puso enérgicamente de relieve que tales formas, en tanto que esquemas de pensamiento, están sometidas a cambios históricos y son capaces de continuar su desarrollo en la historia de las ideas. Pero si nuestra comprensión del mundo depende decisivamente de estas categorías, formas y esquemas de pensamiento, se plantea la cuestión de qué consecuencias tiene esta circunstancia para nuestra concepción de la técnica. Pues bien, Hans Freyer ha destacado en dos ensayos (Über das Dominantwerden technischer Kategorien in der industriellen Gesellschaft (1960) y Die Technik als Lebenswelt, Denkform und Wissenschaft) ${ }^{22}$ que en la sociedad industrial moderna las categorías de progreso, disposición de potencias y factibilidad determinan nuestra acción y nuestra forma de vida.

La categoría más manifiesta y la más utilizada es la de progreso, que, con todo, acompaña a las ciencias y al desarrollo de las técnicas desde Bacon; y aunque desde la primera guerra mundial se han alzado una y otra vez voces críticas contra ella, y precisamente en la filosofía de la técnica, siguió siendo dominante hasta los años sesenta en los países occidentales industrializados, y hoy sigue siéndolo en los países en vías de desarrollo.

En un nivel más profundo se aloja la disposición de potencias, con la que no sólo se piensa en la energía disponible, sino en la disposición de posibilidades en general, lo que supone una inversión completa de la concepción tradicional de la técnica, según la cual la técnica busca los medios adecuados para fines dados y pone en marcha esos medios. Las potencias, desde la toma de corriente hasta el ordenador (cuyo software sólo anticipa como posibilidades ciertos fines muy generales), pasando por el teléfono, que permite llamar y que a uno le llamen, han conducido, en tanto que formas de pensamiento, a una transformación completa de nuestros puntos de vista sobre la técnica, pues ahora buscamos fines, puesto que los medios ya están dados. Esto alcanza tanto al caso trivial de las conversaciones, completamente superfluas, por teléfono móvil a las que asistimos involuntariamente ( Hola, cariño, estoy aquí, en el aeropuerto; enseguida despegamos; hasta luego"), hasta la forzosidad con la

22 Ambos editados en H. FreYER, Gedanken zur Industriegesellschaft, Mainz, v. Hase \& Koehler 1970, págs. 131-144, 145-161. 
que despierta en nosotros la necesidad de un nuevo producto a través de la publicidad, pues el producto fue producido aunque nadie lo echase en falta. Lo que Freyer no pudo intuir hace treinta años es que hoy cualquier PC, y hasta fábricas enteras con robots de producción representan tales potencias libres. No obstante, la disposición de potencias significa al mismo tiempo una ampliación esencial de nuestra libertad de movimientos, precisamente porque podemos determinar nosotros mismos los fines y porque somos nosotros quienes debemos hacerlo.

La tercera forma de pensamiento, la factibilidad, es para Freyer la más central y, al mismo tiempo, la más peligrosa. Este autor la introduce en conexión con una escala (sumamente problemática) de las formas de vida en tanto que estadios de la cultura técnica que André Varagnac introdujo en su obra De la Préhistoire au Monde Moderne. el estadio cultural de los cazadores y recolectores, asignado al reino animal; las culturas de agricultores y pastores, asignadas al reino vegetal; y la cultura moderna desde la revolución industrial, asignada a la materia inerte: hierro, acero, hormigón, plásticos son los materiales dados que (en tanto que algo puramente material) no exigen tampoco consideraciones morales. A la finalidad de la técnica tampoco se opone ningún escrúpulo moral, pues ciertamente existen maltratos contra los animales o las plantas, pero no hay, por ejemplo, maltratos contra la bauxita; hay tortura animal, pero no hay tortura de moléculas. El espíritu técnico sería, pues, libre. Dado que en el ámbito de lo puramente material se han revelado factibles cosas asombrosas, por medio de la producción de nuevos materiales y el desarrollo de nuevos procesos, "la idea de que en el fondo todo debe de ser factible mediante técnicas bien orientadas" ha cobrado "la forma de lo concluyente", se ha convertido en una fórmula propagandística, portadora de ideologías, y ha sido transferida del mundo de las máquinas al mundo social para irrumpir en las técnicas sociales y humanas que "permiten manipular al hombre mismo hasta en sus estructuras impulsivas" ${ }^{23}$. A partir del hacer ha surgido, por totalización, la factibilidad irrestricta. Ésta ha sido criticada con buenas razones (y tenaz y eficazmente por Friedrich Tenbruck en su Kritik der planenden Vernunft ${ }^{24}$ ), pero de ello no nos ocuparemos aquí. Las observaciones de Freyer no se tornan inútiles ni por la unilateralidad del punto partida de

24 F. H. TENBRUCK, Zur Kritik der planenden Vernunft, Freiburg, Alber 1972. 
Varagnac, que desconoce la biotecnología, ni por nuestra sensibilidad hacia los problemas ecológicos, que nos permite hablar con todo derecho de maltrato del medio ambiente, sino que adquieren, en su extensión, cada vez más significado, si bien queda de manifiesto que el inmoralismo de la doctrina de la factibilidad hunde sus raíces en la transferencia de la neutralidad moral de la técnica, en la que se creyó en un principio.

Lo verdaderamente interesante del análisis fenomenológico de Freyer consiste en que interpreta sus formas de pensamiento al mismo tiempo como formas de vida, y por tanto también como formas que dirigen la acción. Tienen poder sobre la historia y sobre la filosofía de la historia. Esto ha sido acentuado a menudo por lo que respecta a la idea de progreso; el nexo entre el desarrollo científico y técnico y un amelioramiento constante no sólo de las condiciones de vida, sino también de la moralidad, se convirtió, desde Bacon, en el hilo conductor de un pathos ilustrado que veía en la cientificación de la técnica y en la tecnificación de todos los ámbitos de la vida el garante por excelencia del progreso de la humanidad; la idea de progreso fue política e históricamente eficaz también en la idea socialista de que sólo hacía falta la sociedad sin clases para asegurar a la larga su eficacia y realización. Pero ya en la forma de pensamiento de la factibilidad se insinúa el peligro que encierra la locura de la factibilidad. En los movimiento tecnocráticos y en toda ideología totalitaria, la forma de pensamiento de la factibilidad es precisamente el hilo conductor de la acción política, nacida de la convicción de que es posible invertir el mundo según los propios fines. Como es sabido, el remedio de Popper se funda en el concepto de una sociedad abierta que, conociendo el riesgo del fracaso, eleva al rango de elemento esencial la crítica abierta tanto a los fines como a los medios, y el control mediante un procedimiento de trial and error. Sin embargo la crítica a la factibilidad suele desarrollarse hoy de otro modo: en la conciencia general aparece más bien como una exhortación y advertencia moralmente fundada, precisamente porque el paralelismo entre el progreso y el hacer se ha cortado y se tiene por verdadera la factibilidad universal. Considérese, por ejemplo, las discusiones públicas sobre la biotecnología, que se llevan a cabo como si el hombre-retorta estilizado mediante técnicas genéticas estuviese a punto de convertirse en realidad.

En cambio, apenas hay críticas a la disposición de potencias, aunque aquí precisamente tiene lugar una inversión total de la relación medios-fines, y de la relación que la humanidad ha tenido con la técnica desde sus comienzos. Al mismo tiempo, esta forma de potencialidad provoca un problema nuevo, 
sumamente característico del presente: el problema de la figura enteramente transformada de la dinámica propia técnica, una dinámica que no se basa en nuevos inventos, sino en la puesta en marcha de medios dados en tanto que potencias, es decir, de encontrar fines para esos medios. Ésta es una característica de la actual técnica sistemática subrayada por Ellul y Landrière ${ }^{25}$ : puesto que los objetos (del automóvil al teléfono) se insertan en una red, surge una tendencia interna de esta red a, por así decirlo, condensarse por sí misma. Nos sometemos a las condiciones del sistema sin haber querido que éste sea tal como es, ya sea al conectar un aparato eléctrico, al telefonear, al navegar en Internet, ya sea en el trabajo en la cadena de montaje o en la decisión acerca de la producción, tomada por un consejo de administración y orientada por el mercado. «Los usuarios sólo actúan en este proceso como medios que contribuyen a proyectar en los sustratos disponibles las posibilidades intrínsecas de la red ${ }^{26}, y$ a hacer, de ese modo, que la red sea cada vez más eficiente, lo que al mismo tiempo tiene como consecuencia cierta autonomía de la red. Esto puede interpretarse también en términos aristotélicos: las posibilidades mismas del sistema tienen, como posibilidad en disposición, una tendencia, una aspiración a la realización.

La disposición de potencias, en una inversión completa de la situación tal como era hasta ahora, convierte a los individuos en medios para la conservación del sistema, al utilizar las potencias y estabilizar así el sistema de forma involuntaria. Ésta es la razón por la cual Ladrière, remontándose a Leibniz, compara los sistemas técnicos con los seres vivos. Pues unos y otros cuidan de su autoconservación y al hacerlo son, en cierto respecto, autónomos. Pero piénsese en la diferencia, destacada por Aristóteles, entre genesis y poiesis. la primera sólo es válida para la vida, la segunda para los artefactos. La poiesis se basa en una idea, en una invención. Ésta, a su vez, exige por una parte un saber, y por otra la valoración de una situación dada como insatisfactoria, valoración vinculada a la representación de una posible situación mejor. La experiencia tiene la función de constatar los nexos que pueden servir como medios para pasar de la primera situación a la segunda.

La dinámica propia de la técnica puede describirse de este modo, y así se comprende por qué esta potencialidad técnica es una posibilidad dinámica

25 J. ElLuL, La Technique ou l'enjou du siècle, Paris, Colin 1954; LANDRIÈRE, op. cit.

26 LANDRIËRE, op. cit. 
aristotélica pese a que todo hacer, toda técnica es siempre poiesis, producción por el hombre, y pese a que la dinámica obtiene su impulso únicamente de la acción de los individuos. A esto corresponde la orientación teleológica atribuida al artefacto en razón de la determinación finalística materializada en él.

La factibilidad por la técnica constituye hoy, indudablemente, uno de los esquemas fundamentales bajo los cuales contemplamos la realidad industrial. Pero a ella se opone, en una gran tensión, otro punto de vista que igualmente puede alegar en su favor el hecho de ser una forma de pensamiento universal del presente: el punto de vista, que hoy se encuentra por todas partes, que modela los procesos temporales como procesos evolutivos ${ }^{27}$. El esquema, tomado de la biología, de mutaciones impredecibles con subsiguientes selecciones fue en un principio trasladado a la ciencia social como darwinismo social, y hoy se encuentra en las teorías lingüísticas del desarrollo de las lenguas, en las teorías de la dinámica de economía, de las ciencias, de la evolución del conocimiento y de la ética. En el desarrollo de la técnica, este esquema significa que una invención impredecible, concebida como mutación e introducida como innovación en el mercado, debe abrirse paso en la distribución entre los compradores en el mismo sentido de una selección contra otros competidores en la struggle for life, o de lo contrario perecerá. De forma análoga sucede en el darwinismo científico: la próxima idea genial de la teoría cuántica es imprevisible; cuando nazca, deberá exponerse a los avatares de la crítica de los especialistas científicos.

En todos los casos citados de transferencia del esquema de la evolución a procesos no biológicos se trata siempre de producciones humanas, de poiesis, pero son modelados con un esquema que Aristóteles habría computado en la genesis, como la forma de desarrollo característica de la vida, con sus propiedades de cambio material, autoconservación y reproducción. La genesis porta en sí su finalidad, mientras que la poiesis lo obtiene del exterior. Pero precisamente esta distinción aristotélica se ve amenazada si se concibe la dinámica de la técnica como evolución. Pues tan pronto como se establece esta forma de pensamiento como absoluta, se asume la imposibilidad de influir sobre el desarrollo de la técnica, porque la dinámica se interpreta como una dinámica propia. Nos

27 Cf. Hans POSER, "Strukturen als Denkformen", in: E. KNOBLOCH (Hg.), Wissenschaft - Technik - Kunst. Interpretationen, Strukturen, Wechselwirkungen (= Gratia 35), Wiesbaden, Harassowitz 1997, 201-214. 
encontramos, pues, frente al dilema de dos formas de pensamiento incompatibles, pero que funcionan simultáneamente: la factibilidad técnica, por un lado, y la evolución de la técnica con su dinámica intangible, por otro lado.

Ambas formas tienen su justificación, pero también sus límites. Y sólo más allá de la determinación de sus límites pueden reconciliarse, de tal modo que los problemas vinculados a ellas no conduzcan necesariamente al temido apocalipsis. Los límites de la factibilidad están ante todo en relación con la posibilidad real de lograr un estado deseado, es decir, con la cuestión de si se trata de una posibilidad meramente lógica, $u$ ontológica, o epistémica. Las utopías y la ciencia-ficción han hecho que los límites se difuminen, ya que no están atadas a las condiciones ontológicas y epistémicas, pues no están obligadas a preguntarse sinceramente si hay una posibilidad de realización. Pero como (de acuerdo con la imagen evolucionista del desarrollo del saber y de la técnica) no es posible decir nada acerca del crecimiento futuro de nuestros conocimientos, las condiciones epistémicas son siempre condiciones históricas y contingentes, mientras que la forma de pensamiento de la factibilidad sugiere precisamente la posibilidad de su transformación. De hecho, los terrores ante el desarrollo de la técnica proceden del supuesto de que la factibilidad está dada, sin importar si se trata del hombre que las técnicas de información vuelven completamente superficial, de los productos biogenéticos de todo tipo o de la colonización de Marte. Pero no es esto propiamente lo que desata los terrores, sino las consecuencias vinculadas a ello, a menudo no intencionadas, desde la pérdida de la libertad e individualidad hasta la preocupación por la vida en Marte, artificial e indigna del hombre, o la destrucción de nuestras condiciones de vida al destruirse el equilibrio ecologico. Los límites de la factibilidad son, indudablemente, los límites que impone el carácter imprevisible de las consecuencias no intencionadas. Pues evitarlas es imposible (si nuestra intención es que una consecuencia determinada no se produzca, éste es precisamente un resultado intencional, no un resultado no intencional). Creer, de acuerdo con la doctrina de la factibilidad, que es posible dominar las consecuencias colaterales perjudiciales y no intencionadas en el caso de que se produjesen, es irresponsable incluso aunque se aceptase la factibilidad de principio, pues entonces podría ser ya demasiado tarde. La ética y la moral siempre han consistido en establecer límites a lo factible. Por eso es irrelevante la cuestión de si la forma de pensamiento de la factibilidad estructura nuestra acción y nuestro pensamiento de manera acertada (una vez que hemos dejado de lado las utopías basadas en ideologías). Se trata, antes bien, de trazar los límites de la factibilidad tal como intenta hacerlo una ética de la responsabilidad. 


\section{Potencia, factibilidad y posibilidad: el problema modal}

Retrospectivamente salta a la vista que las determinaciones centrales tanto del concepto de la técnica como de las formas tecnógenas de pensamiento son conceptos modales: si la técnica es la realización de ideas, es la realización de la posibilidad. Si lo característico del sistema de la técnica es la disposición de potencias, se trata aquí de posibilidades. Y si la factibilidad es la forma de pensamiento central de nuestro presente, se trata igualmente de una posibilidad: la posibilidad de hacer. Estas tres determinaciones son sumamente diferentes $y$, en su diversidad, no pueden ser inmediatamente reunidas mediante las distinciones usuales en teoría modal.

La posibilidad de una idea que cobra realidad en el proceso de realización tiene ante todo el sentido de una posibilidad óntica: la posibilidad de ser, en el sentido de la realizabilidad fáctica. (Todos éstos son, a su vez, conceptos modales. Pero una caracterización no modal es por principio imposible). Pero esta caracterización se queda corta, y al mismo tiempo va demasiado lejos. Se queda corta, porque las posibilidades son algo más que cualesquiera posibilidades ónticas, pues son las formas de solución en el sentido de Dessauer, esto es, medios posibles referidos a un fin. Para poder ser medios deben ser no sólo realizables, sino garantizar además, con su realización, el cumplimiento de una función. Se trata, pues, de posibilidades de cumplimiento de funciones: las formas de solución incluyen este momento teleológico como una necesidad ya en el estado de posibilidad, y ello tanto en el sentido de una condición necesaria como en el sentido de la necesidad física de que en caso de ser realizado, el estado de cosas A conducirá al estado de cosas B, que se desea. Sin embargo, esta caracterización va demasiado lejos, porque se habla de la posibilidad óntica allí donde a nosotros sólo se nos abre un camino que pasa por el saber de relaciones causales, procesos y condiciones iniciales, y una finalidad que pasa por una interpretación valorativa de estados de cosas. Pero esto significa que la posibilidad óntica se funda en una posibilidad epistémica, precisamente en el cumplimiento de las condiciones de conocimiento de un saber de nexos causales y finales. Esto queda de manifiesto cuando nos preguntamos para qué se forman realmente los ingenieros en las escuelas técnicas: no precisamente para construir y utilizar artefactos, procesos y sistemas técnicos, sino para el desarrollo de posibilidades. Un ingeniero piensa en términos de posibilidades (aun cuando su sentido de la realidad le impida reconocerlo). Una ciencia de la construcción proporciona las reglas que designan los medios para 
una transformación de estados de cosas, y adoctrina en la aplicación correcta de estas reglas a los tipos de estados de cosas posibles. La constitución de la posibilidad de la que se trata, en tanto que posibilidad de cumplimiento de una función, se deriva del fundamento de un saber científico-técnico de materiales y de reglas. Es, pues, una posibilidad epistémica que al mismo tiempo debe asegurar, por el tipo de construcción, que es realizable, es decir, que es una posibilidad óntica.

Muy diferentes son las cosas en el caso de las posibilidades dispuestas como potencias. Éstas son manifiestas en su disposición, o más exactamente: vemos un estado de cosas existente como aquello que posibilita otras cosas, sin que éstas otras cosas fuesen primariamente nuestro fin cuando aprovechamos la oferta de potencialidad incorporándonos a la estructura del sistema. En apariencia esto sucede libremente, pues soy yo, ciertamente, quien encarga la instalación de un teléfono. Pero en realidad aceptamos las condiciones del sistema, con el resultado de estabilizar el sistema, dinamizarlo y potenciarlo en su potencialidad. Las posibilidades dispuestas se revelan, pues, como posibilidades dinámicas, pero con dos diferencias frente a las entelequias aristotélicas: en primer lugar, la dinámica es derivada, es decir que procede mediatamente de la intencionalidad del sujeto agente; en segundo lugar, no es teleológica, porque los fines no están prefijados, sino que con la potencialidad sólo está dada una dirección general.

Ahora bien, lo que hemos dicho acerca de la disposición de potencias puede conectarse con las consideraciones anteriores acerca de la posibilidad de cumplimiento de una función, pues ésta ha de ser asegurada también en el caso de la potencialidad: el fin al que apunta el desarrollo de un dispositivo de fabricación, programable (es decir, caracterizado modalmente en esta importante propiedad) y dirigido por ordenador, consiste en la disposición de potencias. Naturalmente, esto es cierto también en su forma más simple, para la producción de un hacha de piedra, pues ésta es tallada no sólo para talar un determinado árbol, sino para talar árboles en general. En este sentido, toda herramienta dispone ciertas potencias. Pero mientras que en el caso de las herramientas su desarrollo tiene lugar a la vista de un ámbito limitado de aplicación, hoy se trata de potencias que por principio son abiertas: el pensamiento técnico ha asumido con ello una forma modal enteramente nueva, pues las posibilidades de cumplimiento de una función ya no se refieren a una máquina concreta, instalada para algún fin perfectamente determinado, sino a la garantía de posibilidades abiertas, potenciales, no fijadas, con vistas a fines que todavía 
han de determinarse, como salta a la vista en cualquier ordenador. Esta nueva forma de pensamiento garantiza, a su vez, los espacios de libertad que se abren, espacios de libertad cuya saturación es impredecible y que por eso mismo hace que cualquier valoración anticipada de la técnica choque con límites insuperables; espacios de libertad, empero, que al mismo tiempo franquean al individuo y a la sociedad la posibilidad, en una amplitud incomparable hasta ahora, de determinarse a sí mismos y de continuar configurando la cultura.

La "factibilidad" es una posibilidad de acción. Vincula, pues, la posibilidad óntica y la posibilidad epistémica, dado el supuesto de la libertad de voluntad y acción del homo faber. en la tradición de Leibniz, Wolff y Kant se plantea la cuestión de en qué consiste el complementum possibilitatis que debe añadirse a la posibilidad ontológica, entendida como puramente conceptual, para que se produzca el acto de devenir real. Para Leibniz consiste en un fiat divino, mientras que Kant rechaza semejante complementum. Pues bien, aquí nos encontramos frente a exactamente el mismo problema: ¿qué se añade a la idea en el hacer, en la realización? Con toda energía hay que aceptarlo: un fiat humano. El supuesto previo de toda técnica es el homo faber, capaz de pensar las posibilidades como posibilidades y como algo completamente nuevo que nunca ha existido, para luego, en su libertad, escoger valorativamente una de esas posibilidades y realizarla.

\section{Perspectivas}

Los problemas que, en tanto que cuestiones filosóficas, se vinculan a la técnica, revelan una extraordinaria amplitud. Aunque aquí hemos puesto entre paréntesis las dificultades particulares de una ética de la técnica, así como las de una epistemología de las ciencias técnicas, se ha mostrado que una filosofía de la técnica hunde sus raíces en ámbitos ontológicos y gnoseológicos, hermenéuticos y de filosofía de la cultura. Tal vez el mejor modo de hacer comprensible el homo faber, para el que ha de ser válida una antropología de la técnica, consista en no detenernos en una teoría de la acción, sino en incorporar esta teoría en una teoría modal: el hombre como el ser capaz de pensar posibilidades, $\mathrm{e}$ incluso posibilidades de posibilidades, de concebirlas de nuevo y de realizarlas. A esta teoría puede añadirse una ética de la técnica entendida como teoría de las razones para la limitación de semejante factibilidad, así como una epistemología de las ciencias técnicas entendida como 
reflexión sobre las condiciones del pensamiento, la fijación, la sistematización y la enseñanza de la factibilidad. Es incierto si, con ello, podrá impedirse la cabalgada de los jinetes del Apocalipsis, pero al menos sabríamos más sobre su camino y es de esperar que también sobre lo que podríamos poner en él para cerrarle el paso, por mor de una técnica más inteligente como condición de la vida y la supervivencia humanas. 